\title{
A Comparison of HIV-Related Risk Factors Between Black Transgender Women and Black Men Who Have Sex with Men
}

\author{
Elizabeth J. Siembida, ${ }^{1, *}$ Lisa A. Eaton, ${ }^{1,2}$ Jessica L. Maksut, ${ }^{1}$ Daniel D. Driffin, ${ }^{2}$ and Robert Baldwin ${ }^{2}$
}

\begin{abstract}
Purpose: Rates of HIV infection among transgender women (TW) are higher than rates observed among men who have sex with men (MSM), and black or African American individuals are at a disproportionately higher risk for HIV than individuals of other races. Limited information, however, is available regarding the needs of black TW and their risk for HIV. Numerous scholarly works and surveillance reports have combined TW with MSM, which has stymied our ability to understand the unique needs of black TW.

Methods: To identify patterns of HIV risk among black TW and black MSM, the current study utilized a crosssectional, convenience sample to compare sociodemographic risk factors, HIV prevention tools, HIV-related risk factors, and psychosocial and sexual risk factors in a sample of 58 black TW and 764 black MSM. Participants were recruited between 2012 and 2014 from Atlanta, GA.

Results: Findings demonstrated that black TW were significantly more likely to report lower educational attainment (odds ratio $[\mathrm{OR}]=0.60,95 \%$ confidence interval $[\mathrm{Cl}]: 0.42-0.85, p=0.005$ ), greater likelihood of being homeless $(\mathrm{OR}=2.49,95 \% \mathrm{Cl}: 1.30-4.78, p=0.006)$, lower HIV testing knowledge ( $\mathrm{OR}=0.66,95 \% \mathrm{Cl}: 0.52-0.83, p=0.001)$, and higher likelihood of having engaged in transactional sex ( $\mathrm{OR}=1.95,95 \% \mathrm{Cl}: 0.99-3.83, p=0.052)$ compared to black MSM.

Conclusions: These findings highlight the need to understand how risk factors for HIV present themselves similarly and differently for both black TW and black MSM, and for HIV prevention programs and interventions to incorporate evidence-based content for each group.
\end{abstract}

Keywords: HIV/AIDS; intersectionality; men who have sex with men; transgender

\section{Introduction}

Men who have sex with men (MSM) historically have been the sociodemographic group most heavily affected by the HIV epidemic in the United States. ${ }^{1,2}$ Recent research, however, suggests that rates of HIV infection among transgender women (TW) are higher than rates observed among $\mathrm{MSM}^{3,4}$ yet few studies have focused exclusively on HIV risk in TW. ${ }^{4}$ Furthermore, research concerning both TW and MSM has found disproportionately higher rates of HIV infection among individuals who identify as black or African
American. ${ }^{1-3}$ The relatively limited data on black TW and the high rates of HIV prevalence among this population suggest that there is an urgent need for further research with this population. ${ }^{5-7}$ Efforts to develop the literature on black TW, and TW more broadly, however, have been hindered by a common practice of including TW within samples of MSM. ${ }^{5-7}$ Although there is a growing literature on risk factors for HIV among TW, there remains a limited yet urgent need to focus on this population. For example, the CDC does not "uniformly collect" data on transgender populations. ${ }^{8}$

\footnotetext{
'Department of Human Development and Family Studies, University of Connecticut, Storrs, Connecticut.

${ }^{2}$ SHARE Project, Atlanta, Georgia.

*Address correspondence to: Elizabeth J. Siembida, PhD, Department of Human Development and Family Studies, University of Connecticut, 348 Mansfield Road, U-1058, Storrs, CT 06269-1058, E-mail: elizabeth.siembida@gmail.com
}

(c) Elizabeth J. Siembida et al. 2016; Published by Mary Ann Liebert, Inc. This Open Access article is distributed under the terms of the Creative Commons License (http://creativecommons.org/licenses/by/4.0), which permits unrestricted use, distribution, and reproduction in any medium, provided the original work is properly credited. 
Based on the limited information regarding TW, a better understanding of their needs is warranted.

Overall, TW experience high rates of sociodemographic risk factors, including homelessness, transactional sex, lower educational attainment, ${ }^{9-12}$ high rates of substance abuse, ${ }^{13,14}$ and physical and sexual violence. ${ }^{13-16}$ Research focused on race/ethnicity and TW has found that black TW report an increased likelihood of drug use, engagement in transactional sex, and drug and alcohol use in the context of sex compared to other ethnic minority TW, ${ }^{9,10,17}$ and ethnic minority TW report lower educational attainment and increased engagement in condomless receptive anal intercourse compared to white TW. ${ }^{12}$

Despite previous research findings concerning black TWs increased risk for HIV compared to black MSM, ${ }^{1-3}$ few studies have compared patterns of HIV risk factors between black TW and black MSM. It is known, however, that minority identities are related to both health promotive and risk taking behavior. According to the intersectionality theoretical framework, ${ }^{18-20}$ multiple identities intersect at microlevels and influence experiences related to privilege and oppression at macrolevels. Intersectionality research highlights the notion that social categories are not independent, but "interdependent and multiconstitutive." ${ }^{21}$ The focus on only one social category (or identity) does not comprehensively explain the disparate and unequal treatment faced by individuals of marginalized groups. Rather, intersectionality posits that unequal and disparate treatment can only begin to be understood when acknowledging the intersection of multiple identities. This framework provides an avenue through which the patterns of HIV risk factors can be contextualized among black TW and black MSM, and highlights the importance of this approach. According to the intersectionality framework, black TW and black MSM may experience risk factors for varying health outcomes differently, yet data from TW are frequently included with MSM or treated as a subcategory of MSM in HIV prevention work.

Consistent with the intersectionality framework, there is some evidence for differing health-related behaviors and sexual risk taking among black MSM and black TW. ${ }^{22}$ For example, race/ethnic minority TW engage in HIV risk taking behaviors (e.g., transactional sex) at higher rates than their race/ethnic minority MSM counterparts. ${ }^{23}$ On the whole, however, there exists limited research on why rates of HIV are higher for black TW compared with black MSM - with both groups experiencing alarmingly high rates of HIV. Sociodemographic variables, health protective behaviors, and internalized beliefs related to HIV prevention may vary across groups and affect rates of HIV among black TW and black MSM. ${ }^{3,4}$ Examining these categories of HIV risk and acknowledging multiple minority identities are critical for developing potential interventions relevant for black TW and black MSM. ${ }^{24}$

\section{Study objectives}

Given the importance of understanding the needs of both black TW and black MSM, the primary objectives of the current study were to examine how HIV risk factors vary between black TW and black MSM. Specifically, we compared (1) sociodemographic variables (i.e., age, education, income, homelessness, sexual orientation, and employment), (2) HIV prevention tools (i.e., ever been tested for HIV, date of last HIV test, heard of pre-exposure prophylaxis (PrEP), taken PrEP, and would take PrEP), (3) HIV-related risk factors (i.e., HIV risk perception, condom use self-efficacy, HIV testing knowledge, and HIV status disclosure), and (4) psychosocial and sexual risk factors (i.e., depression, alcohol use before or during sex, drug use before or during sex, alcohol use, marijuana use, other drug use, condomless anal intercourse, and transactional sex) between black TW and black MSM to identify differences and similarities in patterns of HIV risk taking.

\section{Methods}

Sampling, recruitment, and enrollment

Participants for the current study were recruited in Atlanta, GA, from gay-identified bars, clubs, bathhouses, parks, and street locations; from online classifieds; and on social media (e.g., Facebook, Black Gay Chat, Jack'd) between January 2012 and March 2014. Participants were recruited for an ongoing longitudinal study examining HIV testing counseling interventions. The data presented here come from the baseline survey assessment. Individuals were eligible to participate if they reported engaging in condomless anal sex with a male partner in the past year, male or transgender female gender identity, HIV-negative or unknown status (individuals reporting HIV-positive status were referred to other available studies), and were at least 18 years of age. Before enrollment in the study, potential participants were screened to determine if they fit the study inclusion criteria. Participants recruited at LGBT-friendly locations were screened in-person when recruiters approached individuals as they 
entered the abovementioned venues, and participants recruited online were screened using telephone screening software.

Nine hundred fifty-nine participants screened eligible and attended an in-person appointment at the study research site in Atlanta, GA. During this appointment, participants provided consent and completed a survey assessment that utilized Audio Computer Assisted Interviewing (ACASI) software. Participants were compensated $\$ 30$ for participating in the study. One hundred thirty-seven participants identified as a race/ ethnicity other than black (i.e., white, Latino, Asian, other) and were, therefore, removed from all further analyses. Participants who identified as multirace, including black, were retained in analyses. The final sample for this study included 822 participants. All study procedures were approved by the University of Connecticut Institutional Review Board.

\section{Measures}

Sociodemographic variables. Participants were asked to report on their age, years of education, employment status, income level, race/ethnicity, experiences with homelessness, and sexual orientation (i.e., whether they identified as same gender loving/gay, bisexual, or heterosexual). Our dependent variable, gender identity (i.e., male or transgender female), was established using the two-step method that relies on asking two questions to establish gender identity. ${ }^{25,26}$ Participants were first asked to report what gender they were born (i.e., male or female), and then, participants reported what gender they identify with (i.e., male, female, or transgender [male to female]). Participants were coded as male if they reported being born male and currently identifying as male, or were coded as transgender female if they reported being born male but identifying as female or identifying as transgender (male to female).

\section{HIV prevention tools}

PrEP knowledge and use. PrEP-or taking antiretrovirals (i.e., Truvada) before engaging in sex to prevent HIV infection ${ }^{27}$ - was assessed to gauge the extent to which black TW and black MSM were aware of and using recent HIV prevention advances. Specifically, participants were asked whether they had heard of PrEP, had taken PrEP, and if they would like to take PrEP if provided the opportunity. For each question, participants answered yes or no.

HIV testing history. Participants' experience with HIV testing was assessed with two items. The first item asked if participants had ever been tested for HIV (dichotomous yes or no response), and the second item asked participants the date of their last HIV test (which was reported in number of months between date of assessment and date of test).

\section{HIV-related risk factors}

HIV risk perception. Participants were asked five questions $^{28}$ regarding how much HIV risk they perceived under varying scenarios (e.g., "How risky is anal sex without a condom as the bottom partner with a man you just met who tells you his HIV status is negative?"). Responses ranged from $0=$ no or low risk to $10=$ very high risk and Cronbach's $\alpha=0.80$. An average of the five items was calculated to create an HIV risk perception variable with higher scores indicating greater perceived HIV risk associated with condomless anal sex acts.

Condom use self-efficacy. To measure participants' selfefficacy in negotiating condom use with a partner (e.g., "I feel confident in my ability to discuss condom usage with any partner I might have"), seven items were used from the Condom Use Self-Efficacy Scale (CUSES) by Brafford and Beck. ${ }^{29}$ These seven items were averaged to create a composite condom use self-efficacy variable, and higher scores represented greater condom use selfefficacy. Responses ranged from $1=$ strongly disagree to $6=$ strongly agree and Cronbach's $\alpha=0.90$.

HIV testing knowledge. Participants were asked about their knowledge of HIV testing results. A total of five questions that have been used in previous research were provided to participants with yes or no response options (e.g., "It is possible to test HIV negative but really be HIV positive if someone is recently infected with HIV"). ${ }^{30}$ Participants were given a 1 for a correct answer and a 0 for an incorrect answer. Responses were summed with greater scores indicating higher HIV testing knowledge.

HIV status disclosure. By using three items adapted from Eaton et al., ${ }^{28}$ the current study examined how confident participants were in their ability to discuss HIV status with a new sex partner (e.g., "I am certain that I can ask a new sex partner his HIV status."). Participants responded on a Likert scale ranging from $1=$ strongly disagree to $6=$ strongly agree and Cronbach's $\alpha=0.77$. An average of the three items was calculated, and greater scores indicated higher certainty in being able to discuss HIV status. 
Psychosocial and sexual risk factors

Depressive symptoms. Participants completed the 10item Center for Epidemiological Studies Short Depression Scale (CES-D 10), a screening questionnaire used to measure depressive symptoms (e.g., "In the past week, I was bothered by things that usually don't bother me"). ${ }^{31}$ Participants responded on a Likert scale ranging from $0=$ less than 1 day to $3=5-7$ days, items were reverse coded, if necessary, and Cronbach's $\alpha=0.81$. A sum was calculated to determine the total depression score, and a score of 10 or greater was used as the cutoff for indicating the need for further screening. ${ }^{32}$

Substance use history. Participants were asked two items related to substance use in the context of sex. Participants reported on the number of times they used alcohol before or during sexual activity in the past 3 months, and the number of times they used drugs before or during sexual activity in the past 3 months. Next, participants reported if they had consumed alcohol, marijuana, crack/cocaine, nitrate inhalants, methamphetamines, erectile dysfunction drugs (without a prescription), injected drugs, or other drugs in the past 3 months. Three variables were created from these items: (1) alcohol use, (2) marijuana use, and (3) other drug use (all remaining drugs were combined due to low frequencies). Responses included a dichotomous yes or no.

Sex behaviors and sexual risk taking. Participants were asked to report the total number of times they engaged in condomless anal intercourse with a man in the past 3 months. Transactional sex was also assessed by asking participants if they had ever received money, food, a place to stay, or alcohol/drugs in exchange for sex. Responses included a dichotomous yes or no. Responses were summed across each item, and individuals were given a score of 0 if they had never received any resource in exchange for sex, and a score of 1 if they had received at least one resource in exchange for sex.

\section{Data analysis}

Means, standard deviations, Ns, and percentages were provided for each group on all variables of interest.

Table 1. Sociodemographic Factors Among Black Transgender Women and Black Men Who Have Sex with Men Residing in the Atlanta Metro and Surrounding Areas

\begin{tabular}{|c|c|c|c|c|c|c|c|}
\hline \multirow{4}{*}{ Age } & \multicolumn{2}{|c|}{ BTW $(N=58)$} & \multicolumn{2}{|c|}{ BMSM $(N=764)$} & \multirow[b]{2}{*}{$t$-test } & \multirow[b]{2}{*}{ df } & \multirow[b]{2}{*}{$p$} \\
\hline & $M$ & $S D$ & $M$ & $S D$ & & & \\
\hline & 38.57 & 13.41 & 33.13 & 11.14 & -3.01 & 63.12 & 0.004 \\
\hline & $n$ & $\%$ & $n$ & $\%$ & $\chi^{2}$ & & \\
\hline \multicolumn{8}{|l|}{ Education } \\
\hline Less than high school & 8 & 13.79 & 40 & 5.24 & 34.30 & 5 & $<0.001$ \\
\hline High school & 34 & 58.62 & 227 & 29.71 & & & \\
\hline Some college & 13 & 22.41 & 313 & 40.97 & & & \\
\hline College degree & 2 & 3.45 & 133 & 17.41 & & & \\
\hline Graduate school & 1 & 1.72 & 31 & 4.06 & & & \\
\hline Graduate degree & 0 & 0.00 & 20 & 2.62 & & & \\
\hline \multicolumn{8}{|l|}{ Income } \\
\hline$\$ 0-10,000$ & 46 & 79.31 & 399 & 52.23 & 20.06 & 6 & 0.003 \\
\hline$\$ 11,000-20,000$ & 5 & 8.62 & 144 & 18.85 & & & \\
\hline$\$ 21,000-30,000$ & 2 & 3.45 & 104 & 13.61 & & & \\
\hline$\$ 31,000-40,000$ & 2 & 3.45 & 52 & 6.81 & & & \\
\hline$\$ 41,000-50,000$ & 0 & 0.00 & 33 & 4.32 & & & \\
\hline$\$ 51,000-60,000$ & 0 & 0.00 & 18 & 2.36 & & & \\
\hline$\$ 61,000$ or higher & 1 & 1.72 & 10 & 1.31 & & & \\
\hline Homelessness & 32 & 55.17 & 172 & 22.51 & 32.12 & 1 & $<0.001$ \\
\hline \multicolumn{8}{|l|}{ Sexual orientation } \\
\hline Gay/homosexual/same gender loving & 20 & 34.48 & 358 & 46.86 & 3.49 & 2 & 0.175 \\
\hline Bisexual & 24 & 41.38 & 302 & 39.53 & & & \\
\hline Straight/heterosexual ${ }^{\mathrm{a}}$ & 11 & 18.97 & 97 & 12.70 & & & \\
\hline \multicolumn{8}{|l|}{ Employment status } \\
\hline Unemployed & 49 & 84.48 & 473 & 61.91 & 13.25 & 1 & $<0.001$ \\
\hline Employed & 8 & 13.79 & 291 & 38.09 & & & \\
\hline
\end{tabular}

${ }^{a}$ Men who identified as straight/heterosexual were included in the analysis if they reported at least one instance of engaging in male-to-male sexual contact ( $N=1$ participant was excluded for identifying as straight/heterosexual and reporting no male-to-male sexual contact).

BMSM, black men who have sex with men; BTW, black transgender women. 
Bivariate tests, including independent samples $t$-test with pooled variances, Mann-Whitney $U$ test, or chisquare $\left(\chi^{2}\right)$, were conducted, as appropriate (Tables 1 and 2). Generalized linear modeling was used to conduct multivariable logistic regression to assess the potential differing patterns of risk factors for HIV between black TW and black MSM (Table 3). Generalized linear modeling was utilized because of its flexibility in allowing for non-normal distributions among the included variables.

The following independent variables were analyzed in bivariate analyses with gender identity (black TW or black MSM) as the dependent variable: (1) sociodemographic variables-age, education, income, homelessness, sexual orientation, and employment, (2) HIV prevention tools-ever been tested for HIV, date of last HIV test, heard of PrEP, taken PrEP, and would take PrEP, (3) HIV-related risk factors-HIV risk perceptions, condom use self-efficacy, HIV testing knowledge, and HIV status disclosure, and (4) psychosocial and sexual risk factors-depression, alcohol use before or during sex, drug use before or during sex, alcohol use, marijuana use, other drug use, condomless anal intercourse, and transactional sex.

Table 2. HIV-Related, Psychosocial, and Sexual Risk Factors Among Black Transgender Women and Black Men Who Have Sex with Men

\begin{tabular}{|c|c|c|c|c|c|c|c|}
\hline & \multicolumn{2}{|c|}{ BTW $(N=58)$} & \multicolumn{2}{|c|}{ BMSM $(N=764)$} & \multirow[b]{2}{*}{ Mann-Whitney $U$ test } & \multirow[b]{2}{*}{ df } & \multirow[b]{2}{*}{$p$} \\
\hline & $M$ & $S D$ & $M$ & $S D$ & & & \\
\hline \multicolumn{8}{|l|}{ HIV prevention tools } \\
\hline \multirow[t]{2}{*}{ Months since last HIV test ${ }^{\mathrm{a}}$} & 20.21 & 34.12 & 14.71 & 28.71 & -1.69 & & 0.091 \\
\hline & $n$ & $\%$ & $n$ & $\%$ & $\chi^{2}$ & & \\
\hline Ever been tested for HIV & 42 & 72.41 & 636 & 83.25 & 3.46 & 1 & 0.063 \\
\hline Heard of PrEP & 10 & 17.24 & 184 & 24.08 & 1.26 & 1 & 0.262 \\
\hline Taken PrEP & 1 & 1.72 & 12 & 1.57 & 0.18 & 1 & 0.668 \\
\hline \multirow[t]{2}{*}{ Would take PrEP } & 39 & 67.24 & 624 & 81.68 & 6.49 & 1 & 0.011 \\
\hline & $M$ & $S D$ & $M$ & $S D$ & $t$-test & & \\
\hline \multicolumn{8}{|l|}{ HIV-related risk factors } \\
\hline \multirow[t]{2}{*}{ HIV testing knowledge } & 2.86 & 1.16 & 3.77 & 1.20 & 5.55 & 819 & $<0.001$ \\
\hline & $M$ & $S D$ & $M$ & $S D$ & Mann-Whitney $U$ test & & \\
\hline Condom use self-efficacy & 5.04 & 1.17 & 5.21 & 1.07 & -0.67 & & 0.500 \\
\hline HIV risk perceptions & 7.06 & 2.08 & 7.47 & 1.66 & -0.94 & & 0.357 \\
\hline \multirow[t]{2}{*}{ HIV status disclosure } & 4.45 & 1.60 & 5.10 & 1.21 & -3.02 & & 0.003 \\
\hline & $M$ & $S D$ & $M$ & $S D$ & $t$-test & & \\
\hline \multicolumn{8}{|l|}{ Psychosocial and sexual risk factors } \\
\hline \multirow[t]{2}{*}{ Depressive symptoms (CES-D) } & 10.77 & 6.12 & 10.11 & 6.31 & -0.76 & 819 & .446 \\
\hline & $M$ & $S D$ & $M$ & $S D$ & Mann-Whitney $U$ test & & \\
\hline Condomless anal intercourse & 4.93 & 9.06 & 4.86 & 8.46 & -0.63 & & 0.527 \\
\hline $\begin{array}{l}\text { Number of times alcohol used } \\
\text { before or during sex in past } \\
3 \text { months }\end{array}$ & 5.47 & 10.22 & 5.61 & 10.43 & -0.09 & & 0.928 \\
\hline \multirow{2}{*}{$\begin{array}{l}\text { Number of times drugs } \\
\text { used before or during } \\
\text { sex in past } 3 \text { months }\end{array}$} & 5.93 & 13.12 & 5.43 & 13.57 & -0.40 & & 0.404 \\
\hline & $n$ & $\%$ & $n$ & $\%$ & $\chi^{2}$ & & \\
\hline Engaged in transactional sex & 40 & 68.97 & 334 & 43.71 & 14.83 & 1 & $<0.001$ \\
\hline Used alcohol in past 3 months & 44 & 75.86 & 688 & 90.05 & 9.58 & 1 & 0.002 \\
\hline Used marijuana in past 3 months & 37 & 63.79 & 416 & 54.45 & 2.29 & 1 & 0.131 \\
\hline $\begin{array}{l}\text { Used drugs (except marijuana) } \\
\text { in past } 3 \text { months }\end{array}$ & 31 & 53.45 & 284 & 37.17 & 6.65 & 1 & 0.010 \\
\hline
\end{tabular}

aWithin the study sample, 128 individuals (15.6\%) said they never tested for HIV and therefore this variable could not be calculated for these individuals. Due to this fact, this variable was eliminated from the multivariable model.

CES-D, Center for Epidemiological Studies Short Depression Scale; PrEP, pre-exposure prophylaxis. BMSM, black men who have sex with men; BTW, black transgender women. 
Table 3. Odds Ratios and $95 \%$ Confidence Intervals from the Multivariable Logistic Regression Model with Gender Identity as the Dependent Variable

\begin{tabular}{lcll}
\hline Independent variables & OR & $\mathbf{9 5 \%} \mathrm{Cl}$ & $\boldsymbol{p}$ \\
\hline Age & 1.01 & $(0.98-1.03)$ & 0.631 \\
Education & $\mathbf{0 . 6 0}$ & $\mathbf{( 0 . 4 2 - 0 . 8 5 )}$ & $\mathbf{0 . 0 0 5}$ \\
Income & 0.93 & $(0.67-1.30)$ & 0.681 \\
Homelessness & $\mathbf{2 . 4 9}$ & $\mathbf{( 1 . 3 0 - 4 . 7 8 )}$ & $\mathbf{0 . 0 0 6}$ \\
Employment status & 1.67 & $(0.69-4.02)$ & 0.252 \\
Ever been tested for HIV & 0.87 & $(0.44-1.71)$ & 0.688 \\
Would take PrEP & 0.53 & $(0.27-1.02)$ & 0.058 \\
HIV testing knowledge & $\mathbf{0 . 6 6}$ & $\mathbf{( 0 . 5 2 - 0 . 8 3 )}$ & $\mathbf{0 . 0 0 1}$ \\
HIV status disclosure & 0.91 & $(0.75-1.11)$ & 0.351 \\
Engaged in transactional sex & $\mathbf{1 . 9 5}$ & $\mathbf{( 0 . 9 9 - 3 . 8 3 )}$ & $\mathbf{0 . 0 5 2}$ \\
Used alcohol in last 3 months & $\mathbf{0 . 3 3}$ & $\mathbf{( 0 . 1 6 - 0 . 7 0 )}$ & $\mathbf{0 . 0 0 4}$ \\
Used drugs (except pot) in last 3 months & 0.94 & $(0.48-1.85)$ & 0.855 \\
\hline
\end{tabular}

Variables found to be significantly associated with gender identity are bolded.

Black MSM were used as the reference category in the multivariable logistic regression analysis.

$\mathrm{Cl}$, confidence interval; $\mathrm{OR}$, odds ratio.

Independent variables in the bivariate analyses that were found to be significantly different between black TW and black MSM at the $p<0.10$ level were included in the multivariable analysis. In the multivariable analysis, variables were entered simultaneously and odds ratios (ORs) and their 95\% confidence intervals (CIs) were calculated. For the multivariable analysis, $p<0.05$ was used to define statistical significance. Data analyses were completed between June 2015 and August 2015, and less than $5 \%$ of data were missing for any given variable. We used PASW Statistics, version 19.0 (SPSS, Inc., IBM, Somers, NY) for all analyses.

\section{Results}

Sociodemographic variables

Of the total sample, 58 participants (7\%) identified as black TW and 764 (93\%) identified as black MSM. Black TW were significantly more likely to be older $[t(63.12)=-3.01, p=0.004]$, less educated $\left[\chi^{2}(5)=34.30\right.$, $p<0.001]$, unemployed $\left[\chi^{2}(1)=13.25, p<0.001\right]$, have lower incomes $\left[\chi^{2}(6)=20.06, p=0.003\right]$, and experience homelessness $\left[\chi^{2}(1)=32.12, p<0.001\right]$ compared with black MSM (Table 1).

\section{HIV prevention tools}

Overall, 678 respondents (82.5\%) reported having been tested for HIV at least once in their lifetime. Bivariate analyses found that black TW were less likely to have been tested for HIV $\left[\chi^{2}(1)=3.46, p=0.063\right]$, and for those participants who reported having an HIV test, more time had passed since black TW had an HIV test compared to black MSM (Mann-Whitney $U=$ $-1.69, p=0.091)$. Although there were few differences between black TW and black MSM on their knowledge and use of PrEP, black TW were significantly less likely to want to use PrEP if they had access to it compared with black MSM $\left[\chi^{2}(1)=6.49, p=0.011\right]$ (Table 2).

\section{HIV-related risk factors}

On average, participants reported an HIV risk perception score of $7.45(S D=1.70)$, indicating medium to high levels of risk, and there was no statistically significant difference on HIV risk perception between black TW and black MSM. The mean condom use selfefficacy score for the total sample was 5.20 ( $S D=$ 1.08 ), and there was no statistically significant difference on condom use self-efficacy between black TW and black MSM. Black TW were significantly less likely to feel comfortable having discussions about HIV status with a new partner (Mann-Whitney $U=-3.02$, $p=0.003$ ) compared with black MSM. When asked about HIV testing knowledge, black TW scored significantly lower, indicating less understanding about HIV testing and HIV test results than black MSM $[t(819)=5.55, p<0.001]$ (Table 2).

\section{Psychosocial and sexual risk factors}

Overall, 389 (47.3\%) participants scored 10 and above on the CES-D 10, the threshold score indicating the need for further screening for depression. There were no significant differences on CES-D 10 scores across groups. Participants reported drinking alcohol before or during sex 5.60 times $(S D=10.41)$ in a 3 -month period, and using drugs before or during sex 5.46 times $(S D=13.52)$ in a 3-month period. Seventysix percent of black TW and $90.1 \%$ of black MSM reported drinking alcohol in the past 3 months, $63.8 \%$ of black TW and $54.5 \%$ of black MSM reported using marijuana in the past 3 months, and $53.5 \%$ of black TW and $37.2 \%$ of black MSM reported using any drugs (except marijuana) in the past 3 months. Black TW were significantly more likely to report using any drug (except marijuana) in the last 3 months $\left[\chi^{2}(1)=\right.$ 6.65, $p=0.010$ ] and significantly less likely to report drinking alcohol in the last 3 months $\left[\chi^{2}(1)=9.58\right.$, $p=0.002$ ] compared with black MSM.

Although all participants in the study reported at least one act of condomless anal intercourse in the last year as part of the study's inclusion criteria, there was no significant difference in the total number of 
condomless anal intercourse acts reported by black TW and black MSM. Sixty-eight percent of black TW reported ever engaging in transactional sex versus $43.7 \%$ of black MSM $\left[\chi^{2}(1)=14.83, p<0.001\right]$ (Table 2$)$.

\section{Multivariable logistic regression model}

After identifying HIV risk factors in the bivariate analysis that were associated with gender identity at the $p<0.10$ level, these risk factors were examined in a multivariable model. Multiple variables were found to be significantly associated with gender identity. Specifically, in the multivariable model, lower educational attainment $(\mathrm{OR}=0.60,95 \% \mathrm{CI}: 0.42-0.85, p=0.005)$, higher likelihood of being homeless $(\mathrm{OR}=2.49,95 \%$ CI: $1.30-4.78, p=0.006)$, lower understanding of HIV test results $(\mathrm{OR}=0.66,95 \% \mathrm{CI}: 0.52-0.83, p=0.001)$, and higher likelihood of having engaged in transactional sex $(\mathrm{OR}=1.95,95 \% \mathrm{CI}$ : $0.99-3.83, p=0.052)$ were associated with identifying as black TW. Black MSM were more likely to have consumed alcohol in the last 3 months $(\mathrm{OR}=0.33,95 \% \mathrm{CI}: 0.16-0.70$, $p=0.004)$ than black TW.

\section{Discussion}

Overall, the extent to which black TW and black MSM experience disadvantage and, more specifically, risk factors associated with HIV transmission, is alarming. Both black TW and black MSM reported sociodemographic factors and behaviors associated with increased risk for HIV in the current study, but varying patterns of HIV risk were found among these two populations. It is important to consider these findings in the context of the intersectionality framework, as the greater psychosocialrelated burdens observed among TW in the current study highlight the need to acknowledge and understand the intersection of the multiple minority identities. Specifically, this approach is important because it can be used to guide how outreach and linkage to care programs (and other related engagement programs) are shaped for addressing the unique needs of each population. ${ }^{18-20}$ For example, TW may experience uncertainty in linking to HIV prevention care when much of the programming is targeted to MSM. Without acknowledging multiple identities, there is the potential for overlooking factors that are critical to the success of improving the social conditions of each minority group. Furthermore, the findings are consistent with previous research focused on $\mathrm{TW}^{33}$ and this study is among the first to compare the patterns of HIV risk factors between black TW and black MSM.
Although data from the current study suggest that both black MSM and black TW face numerous sociodemographic- and psychosocial-related burdens, black TW reported lower education attainment and higher rates of homelessness and engagement in transactional sex. Given what is understood about the relationships between sociodemographic risk factors for HIV and actual HIV transmission rates, ${ }^{34-36}$ these disparities place black TW at greater risk for HIV transmission. Homelessness, in particular, is a notable factor for increasing likelihood of HIV transmission as this relationship is thought to exist through the reliance on transactional sex for getting basic needs met (e.g., shelter). As a marginalized group with intersecting minority identities, difficulty in securing employment (e.g., via discrimination, prejudice, and bias against gender minority populations), and, therefore, experiencing an inability to gain basic living necessities are likely important drivers in their engagement in transactional sex compared to black MSM. ${ }^{37,38}$

The stigma related to homelessness may also act as a social barrier to seeking out healthcare, and, potentially, to exposure to sexual networks where HIV prevalence is elevated. ${ }^{39,40}$ There exist, however, very limited data on these disparities specifically related to TW. Prior work among race minority TW has found that experiencing structural barriers, including homelessness, has been associated with a greater likelihood of experiencing transphobic victimization (emotional, physical, and sexual abuse due to being transgender) and engaging in sexual risk taking. ${ }^{41}$ Research with black MSM has also found that unstable housing is associated with condomless sex with a serodiscordant partner. ${ }^{42}$ In the same sample of black MSM, lower educational attainment was associated with condomless sex with a nonmain sex partner. ${ }^{42}$ On the whole, establishing a comprehensive understanding of multilevel factors related to HIV transmission for both black TW and MSM populations is imperative for HIV prevention and treatment, and the accumulation of these factors likely places black TW at higher risk for HIV infection. ${ }^{43}$

The lack of access to basic living necessities also impacts black TWs linkage to healthcare and HIV prevention knowledge. ${ }^{44,45}$ With lower rates of accessing HIV test results and lowered interest in using prevention tools such as PrEP, black TW remain at an increased risk for HIV compared to black MSM. Facilitating access to HIV testing and counseling is an urgent first step in reducing the burden of HIV among black 
TW. Attaining this goal, however, is reliant on HIV prevention programs, including engagement strategies and intervention components focused on the unique needs of black TW. ${ }^{46}$

The high rate of depressive symptoms reported among both black TW and black MSM in the current study is noteworthy. Reisner et al. ${ }^{47}$ highlight the relationship between depression and increased risk for HIV among black MSM, and the need to address screening and treatment for depression before engagement in further intervention programs. Slightly less than half of the sample reported depressive symptoms that indicate the need for further screening. Examining this finding in combination with the previous findings regarding sociodemographic risk factors among black TW and black MSM reinforces the urgency of meeting the basic needs of black TW and black MSM. Failing to meet these needs likely prohibits the implementation of recent advances in prevention options (e.g., PrEP).

\section{Limitations}

The findings from the current study should be interpreted in light of their limitations. Specifically, the data presented in this study were collected with a cross-sectional approach, and therefore, any conclusions based on causality or the directions of specific relationships cannot be made. In addition, the participants in the current study were recruited from LGBTfriendly venues or through online venues targeted toward LGBT populations, and participants reported condomless anal sex in the past year as part of eligibility screening. The use of these sampling techniques may bias the conclusions of the study. Specifically, the study does not include black TW who only have sex with women, individuals who do not engage in condomless anal sex, or black TW and black MSM who frequent other venues. The lack of representation of these individuals in the current study may underrepresent HIV risk factors among all black TW and black MSM, and likely affects our observed patterns of risk taking. Finally, all of the participants in the current study lived in the Atlanta metropolitan and surrounding areas, and their experiences may not generalize to other regions or rural populations of black TW and black MSM.

\section{Conclusion}

The current study highlights the need to consider black TW separate from black MSM when examining HIV risk in these populations. Likewise, when creating HIV prevention programs, researchers/commu- nity agencies need to carefully consider the audience they are targeting and evaluate the needs of programming specific to their target population. As posited by intersectionality theory, the multiple minority statuses of black TW must be given strong consideration when developing outreach efforts and intervention content-doing so will ensure that HIV prevention and treatment programs appeal to and reach the needs of black TW.

\section{Acknowledgments}

This project was supported by the National Institute of Mental Health grant R01MH094230 and by the National Institute of Nursing Research grant R01NR013865.

\section{Author Disclosure Statement}

No competing financial interests exist.

\section{References}

1. Prejean J, Song R, Hernandez A, et al. Estimated HIV incidence in the United States, 2006-2009. Lee V, ed. PLoS One. 2011;6:e17502.

2. Millett GA, Peterson JL, Wolitski RJ, Stall R. Greater risk of HIV infection of Black men who have sex with men: a critical literature review. Am J Public Health. 2006;96:1007-1019.

3. Herbst JH, Jacobs ED, Finlayson TJ, et al. Estimating HIV prevalence and risk behaviors of transgender persons in the United States: a systematic review. AIDS Behav. 2008;12:1-17.

4. Baral SD, Poteat T, Strömdahl S, et al. Worldwide burden of HIV in transgender women: a systematic review and meta-analysis. Lancet Infect Dis. 2013;13:214-222.

5. Brito MO, Hodge D, Donastorg Y, et al. Risk behaviours and prevalence of sexually transmitted infections and HIV in a group of Dominican gay men, other men who have sex with men and transgender women. BMJ Open. 2015;5:e007747.

6. Castillo R, Konda KA, Leon SR, et al. HIV and sexually transmitted infection incidence and associated risk factors among high-risk MSM and male-tofemale transgender women in Lima, Peru. J Acquir Immune Defic Syndr. 2015;69:567-575.

7. Lee SW, Deiss RG, Segura ER, et al. A cross-sectional study of low HIV testing frequency and high-risk behaviour among men who have sex with men and transgender women in Lima, Peru. BMC Public Health. 2015;15:408.

8. CDC. HIV/AIDS [Internet]. Atlanta, GA: Centers for Disease Control. HIV Among Transgender People. April 18, 2016 [cited 2016 May 16]; [about 3 screens]. Available at: www.cdc.gov/hiv/group/gender/transgender/index.html (accessed May 10, 2016)

9. Garofalo R, Deleon J, Osmer E, et al. Overlooked, misunderstood and atrisk: exploring the lives and HIV risk of ethnic minority male-to-female transgender youth. J Adolesc Health. 2006;38:230-236.

10. Nemoto T, Sausa LA, Operario D, Keatley J. Need for HIV/AIDS education and intervention for MTF transgenders: responding to the challenge. J Homosex. 2006;51:183-201.

11. Wilson EC, Garofalo R, Harris RD, et al. Transgender female youth and sex work: HIV risk and a comparison of life factors related to engagement in sex work. AIDS Behav. 2009;13:902-913.

12. Wilson EC, Chen $Y$, Arayasirijul S, et al. Differential HIV risk for racial/ethnic minority trans*female youth and socio-economic disparities in housing, residential stability and education. Am J Public Health. 2015;105:e41-e47.

13. Clements-Nolle K, Marx R, Guzman R, Katz M. HIV prevalence, risk behaviors, health care use, and mental health status of transgender persons: implications for public health intervention. Am J Public Health. 2001;91:915-921.

14. Xavier JM, Bobbin M, Singer B, Budd E. A needs assessment of transgendered people of color living in Washington DC. Int J Transgend. 2005;8:31-47. 
15. Kenagy GP, Bostwick WB. Health and social service needs of transgender people in Chicago. Int J Transgend. 2005;8:57-66.

16. Lombardi EL, Wilchins RA, Priesing D, Malouf D. Gender violence: transgender experiences with violence and discrimination. J Homosex. 2001;42:89-101.

17. Nemoto T, Operario D, Keatley J, et al. HIV risk behaviors among male-tofemale transgender persons of color in San Francisco. Am J Public Health. 2004;94:1193-1199.

18. Crenshaw K. Demarginalizing the intersection of race and sex: a Black feminist critique of antidiscrimination doctrine, feminist theory and antiracist politics. Univ Chicago Legal Forum. 1989;140:139-167.

19. Meyer D. An intersectional analysis of lesbian, gay, bisexual, and transgender (LGBT) people's evaluations of anti-queer violence. Gend Soc. 2012;26:849-873.

20. McCall L. The complexity of intersectionality. Signs (Chic). 2005;30: 1771-1800.

21. Bowley L. The problem with the phrase women and minorities: intersectionality - an important theoretical framework for public health. Am J Public Health. 2012;102:1267-1273.

22. Miller LR, Grollman EA. The social costs of gender nonconformity for transgender adults: implications for discrimination and health. Sociol Forum. 2015;30:809-831.

23. Sanchez T, Finlayson T, Murrill C, et al. Risk behaviors and psychosocial stressors in the New York City house ball community: a comparison of men and transgender women who have sex with men. AIDS Behav. 2010;14:351-358.

24. Hendricks ML, Testa RJ. A conceptual framework for clinical work with transgender and gender nonconforming clients: an adaptation of the minority stress model. Prof Psychol Res Pract. 2012;43:460-467.

25. The GenIUSS Group. Best Practices for Asking Questions to Identify Transgender and Other Gender Minority Respondents on PopulationBased Surveys. Los Angeles, CA: The Williams Institute, 2014.

26. Tate CC, Ledbetter JN, Youssef CP. A two-question method for assessing gender categories in the social and medical sciences. J Sex Res. 2013;50:767-776.

27. Grant RM, Lama JR, Anderson PL, et al. Preexposure chemoprophylaxis for HIV prevention in men who have sex with men. N Engl J Med. 2010;363:2587-2599.

28. Eaton LA, Kalichman SC, Cain DN, et al. Serosorting sexual partners and risk for HIV among men who have sex with men. Am J Prev Med. 2007;33:479-485.

29. Brafford LJ, Beck KH. Development and validation of a condom selfefficacy scale for college students. J Am Coll Health. 1991;39:219-225.

30. Eaton LA, Driffin DD, Bauemeister J, et al. Minimal awareness and stalled uptake of pre-exposure prophylaxis (PrEP) among at risk, HIV-negative, Black men who have sex with men. AIDS Patient Care STDS. 2015;29:423430.

31. Irwin M, Artin KH, Oxman MN. Screening for depression in the older adult: criterion validity of the 10-item Center for Epidemiological Studies Depression Scale (CES-D). Arch Intern Med. 1999;159:1701-1704.

32. Björgvinsson T, Kertz SJ, Bigda-Peyton JS, et al. Psychometric properties of the CES-D 10 in a psychiatric sample. Assessment. 2013;20:429-436.

33. Brennan J, Kuhns LM, Johnson AK, et al. Syndemic theory and HIV-related risk among young transgender women: the role of multiple, co-occurring health problems and social marginalization. Am J Public Health. 2012; 102:1751-1757.

34. Sumartojo E. Structural factors in HIV prevention: concepts, examples, and implications for research. AIDS. 2000;14(Suppl 1):S3-S10.

35. Gupta GR, Parkhurst JO, Ogden JA, et al. Structural approaches to HIV prevention. Lancet. 2008;372:764-765.

36. Fuqua V, Chen YH, Packer T, et al. Using social networks to reach Black MSM for HIV testing and linkage to care. AIDS Behav. 2012;16:256-265.

37. Fang J, Yang Q, Ayala C, Loustalot F. Disparities in access to care among US adults with self-reported hypertension. Am J Hypertens. 2014;27: 1377-1386.

38. Morales LS, Cunningham WE, Galvan FH, et al. Sociodemographic differences in access to care among hispanic patients who are HIV infected in the United States. Am J Public Health. 2004;94:1118-1121.

39. Takahashi LM. The socio-spatial stigmatization of homelessness and HIV/ AIDS: toward an explanation of the NIMBY syndrome. Soc Sci Med. 1997;45:903-914.
40. Bobashev GV, Zule WA, Osilla KC, et al. Transactional sex among men and women in the south at high risk for HIV and other STIs. J Urban Health. 2009;86(Suppl 1):32-47.

41. Raiford JL, Hall GJ, Taylor RD, et al. The role of structural barriers to risky sexual behavior, victimization and readiness to change HIV/STI-related risk behavior among transgender women. AIDS Behav. 2016; [Epub ahead of print]; DOI: 10.1007/s10461-016-1424-8.

42. Mimiaga MJ, Reisner SL, Cranston K, et al. Sexual mixing patterns and partner characteristics of Black MSM in Massachusetts at increased risk for HIV infection and transmission. J Urban Health. 2009;86: 602-623.

43. Oldenburg CE, Perez-Brumer AG, Reisner SL, Mimiaga MJ. Transactional sex and the HIV epidemic among men who have sex with men (MSM): results from a systematic review and meta-analysis. AIDS Behav. 2015;19:2177-2183.

44. Reback CJ, Ferlito D, Kisler KA, Fletcher JB. Recruiting, linking, and retaining high-risk transgender women into HIV prevention and care services: an overview of barriers, strategies, and lessons learned. Int $J$ Transgend. 2015;16:209-221.

45. Sevelius JM, Patouhas E, Keatley JG, Johnson MO. Barriers and facilitators to engagement and retention in care among transgender women living with human immunodeficiency virus. Ann Behav Med. 2014;47: 5-16.

46. Pettifor A, Nguyen NL, Celum C, et al. Tailored combination prevention packages and PrEP for young key populations. J Int AIDS Soc. 2015;18(Suppl 1):19434.

47. Reisner SL, Mimiaga MJ, Skeer M, et al. Clinically significant depressive symptoms as a risk factor for HIV infection among black MSM in Massachusetts. AIDS Behav. 2009;13:798-810.

Cite this article as: Siembida EJ, Eaton LA, Maksut JL, Driffin DD, Baldwin R (2016) A comparison of HIV-related risk factors between black transgender women and black men who have sex with men, Transgender Health 1:1, 172-180, DOI: 10.1089/trgh.2016.0003.

$\begin{aligned} & \text { Abbreviations Used } \\ & \text { ACASI }=\text { Audio Computer Assisted Interviewing } \\ & \text { BMSM }=\text { black men who have sex with men } \\ & \text { BTW }=\text { black transgender women } \\ & \text { CES-D } 10=\text { Center for Epidemiological Studies Short } \\ & \quad \text { Depression Scale } \\ & \mathrm{Cls}=\text { confidence intervals } \\ & \mathrm{CUSES}=\text { Condom Use Self-Efficacy Scale } \\ & \mathrm{MSM}=\text { men who have sex with men } \\ & \mathrm{ORs}=\text { odds ratios } \\ & \mathrm{PrEP}=\text { pre-exposure prophylaxis } \\ & \mathrm{TW}=\text { transgender women }\end{aligned}$

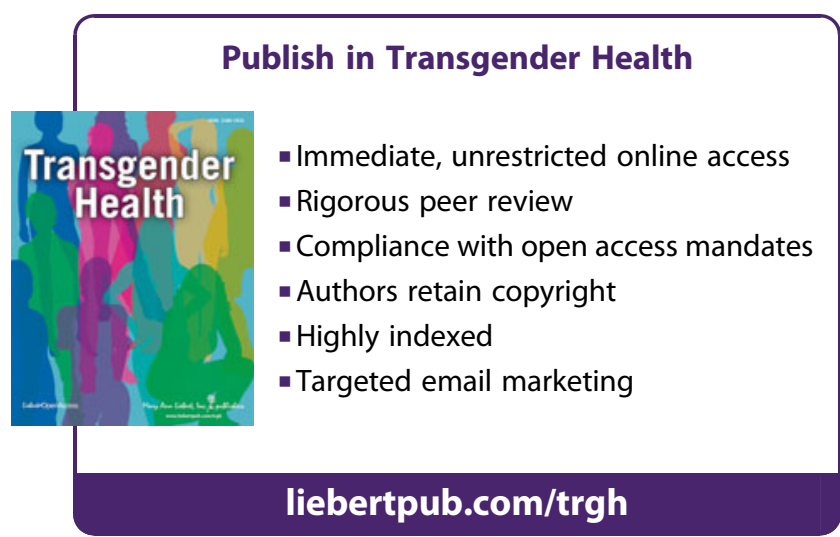

